\title{
Theory of Quantum Relativity
}

\author{
Sudhanva Joshi \\ Sies College of Arts, Commerce and Science, Mumbai, India \\ Email: Sudhanvaj@sies.edu.in
}

How to cite this paper: Joshi, S. (2016) Theory of Quantum Relativity. Journal o1 Quantum Information Science, 6, 249-262. http://dx.doi.org/10.4236/jqis.2016.64016

Received: April 17, 2016

Accepted: November 13, 2016

Published: November 16, 2016

Copyright $(\odot 2016$ by author and Scientific Research Publishing Inc. This work is licensed under the Creative Commons Attribution International License (CC BY 4.0).

http://creativecommons.org/licenses/by/4.0/

(c) (i) Open Access

\begin{abstract}
In this paper, I have studied the properties of atomic and molecular world along with general and special theories of relativity. This is an attempt to merge Gravity into the standard model in order to complete the Grand Unification Theory. The merger of gravity into the other forces i.e. electromagnetic, strong and weak nuclear forces should be well defined and in the boundaries of Gauge Group theory. The Lorentz transformations used in the theory too are invariant under $\mathrm{SU}(2)$ type of space. The relative force exerted on two separate quantum systems is also discussed along with Dark matter and Dark energy at a quantum level. I have also tried to solve the Banach-Tarski theorem by applications of Heisenbergs Uncertainty principle in the later part of the paper. Detailed particle Chirality in standard model is redefined to fit in the criterion of operators used in the same process. Possible existence of a new quasi particle is also included in the paper along with its properties.
\end{abstract}

\section{Keywords}

Gauge-Group Theory, String Theory, G-Bosons, Quantum Relativity

\section{Introduction}

The nature of matter has baffled physicists for a long time now. The very base of standard model is an assumption of gravity being a bosonic force. The task of merging gravity into the standard model is almost impossible as gravity differs from other forces in various aspects such as its spin and its Hamiltonian invariance under appropriate gauge group $\mathrm{SU}(2) \times \mathrm{U}(1)$ [1] even though some progress has been made especially after discovery of Gravitational waves. So far there has never been a conclusive proof of existence of spin $=2$ particle as it barely interacts with fermionic matter and even weakly charged matter [2]. There needs to be a backup hypothesis for existence of gravitons (either that or standard model should accept the Hamiltonian constraints). In this paper, the Quantum Field theory is discussed in Section 2. Section 3 intricately 
discusses basic and advanced interpretation of Hawkings Grand unification theory and also the Wheeler-Dewitt metric. Section 4 talks about the standard model in general and detailed Lagrangians of Higgs-Mechanism; it also further discusses Quantum coupling and Planck scale in standard model. Section 5 is main part of this enterprise; it discusses about my concept regarding quantum chirality and quantum relativity. In the 2nd part of this section, quantum chirality is merged with relativity to make relativistic quantum chirality. In the final section, scopes of Quantum Relativity are discussed. It is sort of implementation of quantum theory of relativity. Here I have introduced condensing phasor equations which is to simplify the mass and momenta problem while solving Kahler-Einstein statistics [3]. The paper also covers existence of a new quasi particle with some properties like Polarons using Hubbard Stratonovich transformations.

\section{Quantum Field Theory and Its Generators}

The formation of universe is a cataclysmic process which involves a theoretical framework of point like one dimensional string [4]. String theory discusses it further. The string theory is aided by Conformal Field Theory (CFT) which is QFT invariant under conformal transformations. There are two variants of CFT: Euclidean which is extension of statistical mechanics and Lorentzian which is extension of quantum field theory [5]. The above transformations are related by Wick Rotation [6]. For example, considering CFT variant group on Riemannian sphere is Mobius Transformation. It is a projective linear group $\operatorname{PSL}(2, \mathrm{C})$. The generators are indexed as:

$$
l_{n}=\int_{z=0} \frac{\mathrm{d} z}{d \pi i} Z^{n+1} T_{z z}
$$

$T_{z z}$ is the isotropic topological component of energy-momentum tensor of field theory. The free scalar field generator is derived as:

$$
t_{z z}=\frac{1}{2}\left(\partial_{z z} \varphi\right)^{2}
$$

The Quantum field theory is theory of indexing generators in quantum topology. The most prominent QFT being Gauge theory. It successfully describes the motion and annihilation of subatomic particles. Here, particle interactions are discussed with help of Feynman diagrams. Considering earlier discussed CFT, The Fourier transformations on Euclidean space will be:

$$
\check{f}(\xi)=\int_{\mathbb{R}}^{1} f(x) \mathrm{e}^{-2 \pi i x \cdot \xi} \mathrm{d} x
$$

where $x$ and $\xi$ are n-dimensional operators. The Fourier transformation on Lorentzian space will be nonexistent as general function will be discontinuous. To sum it up, at quantum level physics behaves entirely differently by virtue of the laws discussed above.

\section{The Grand Unification Theory}

The attempt for unification started back in later part of 19th century by James Clark 
Maxwell. Earlier, Hans Christian Oersted stated that electric currents produce magnetic fields. Until then electric and magnetic fields were considered as totally different phenomena. Then in $1864 \mathrm{~J}$. C Maxwell Published Dynamics of Electromagnetic field theory. In the same paper, Maxwell stated the wave equation of electromagnetic fields and also the velocity of light. The very first modern attempt was with unified Electroweak Theory [7]. Mathematically this theory comes under $\mathrm{SU}(2) \times \mathrm{U}(1)$ Gauge group. There are 4 types of gauge bosons: $-\mathrm{W}(+,-)$ boson, $\mathrm{Z}$ boson, photon. The fifth one is scalar field boson or the so dubbed God particle Higgs boson. The other vector bosons are produced by symmetry breaking $\mathrm{SU}(2) \times \mathrm{U}(1)$ as casimir group and lie group. This kind of symmetry breaking results in combining of $Z$ boson and photon is given as:

$$
\left(\frac{\gamma}{Z}\right)=\left(\begin{array}{cc}
\cos \theta_{w} & \sin \theta_{w} \\
-\sin \theta_{w} & \cos \theta_{w}
\end{array}\right)\left(\frac{B}{W 3}\right)
$$

where $w$ is mixing angle. Also, the final Lagrangian is represented as sub-derivative of Yukawa interactions as:

$$
\mathcal{L}_{Y}=-\sum_{f} \frac{g m_{f}}{2 m_{w}} \overline{f f} \bar{H}
$$

The Electroweak unified theory became a template of modern GUT. The more modern approach includes Gravity in unification of all forces [8]. So far there is no success so as to unify gravity even though attempts have been made. Most Promising of them are Loop Quantum Gravity and String Theory. In LQG, the gravity is quantized in 4 dimensions (as Lorentz group transforms into 2 Spinors) by canonical formalism. Fourier transformation is used to convert to loop variables. Furthermore, the trivial Wheeler-Dewitt equation can be solved using states spanned by Knots and the YangBaxter equation [9]. The canonical gravity is represented by 3-metric tensor. The spacetime is foliated as Knots. Statistically, the equation is derived as:

$$
g_{v v} \mathrm{~d} x^{v} \mathrm{~d} x^{v}=\left(-N^{2}+\beta_{\kappa} \beta_{\kappa}\right) \mathrm{d} t^{2}+2 \beta_{\kappa} \mathrm{d} x^{\kappa}+\gamma_{i j} \mathrm{~d} x^{i} \mathrm{~d} x^{j}
$$

In that equation the Weyl gravitational field is convergent. Its Hamiltonian is a constraint as:

$$
H=\frac{1}{2 \sqrt{\gamma}} G_{i j} \pi^{i j} \pi^{k l}-\sqrt[3]{\gamma} R
$$

Here $\gamma=\operatorname{det}\left(\gamma_{i j}\right)$ and $G_{i j k l}=\left(\gamma_{i k} \gamma_{j l}+\gamma_{i l} \gamma_{j k}-\gamma_{i j} \gamma_{k l}\right)$ is the Wheeler-DeWitt metric. The path-integral derivation includes Gravitational action in the Euclidean Quantum Gravity paradigm and is given as:

$$
Z=\int_{c}^{1} \mathrm{e}^{-I\left[g_{v v}, \phi\right]} D_{G} D_{\phi}
$$

Here, the Hamiltonian constraint is obtained as;

$$
\frac{\delta I_{E H}}{\delta N}=0
$$

where $I_{E H}$ is Einstein-Hilbert action. The Lagrangian $(N)$ is in the lapse phase and its Wheeler-Dewitt metric can be derived as: 


$$
\frac{\delta Z}{\delta N}=0=\int \frac{\delta I\left[g_{v v}, \phi\right]}{\delta N} \mid \sum \exp \left(-I\left[g_{v v}, \phi\right]\right) D g D \phi
$$

To sum it up, Wheeler-DeWitt equation simply states as:

$$
\hat{H}=|x\rangle|x\rangle
$$

where $\hat{H}|x\rangle$ is the Hamiltonian constraint in quantized general relativity and $|x\rangle$ stands for the wave function of the universe. Taking the String Theory, most important is super Yang-Mills scattering theory in 4 dimensions (as the null vectors can be split into 2 spinors). The approach taken here is that mass less theories have ConformalInvariance in Space time but it can be transformed into dual momentum space using a Fourier transformation where amplitudes become loop variables. The closure of dual momentum space symmetries is an infinite dimensional Yangian symmetry which too uses the yang-Baxter equation.

$$
\left\{t_{i j}^{p}+1, t_{k l}^{q}\right\}-\left\{t_{i j}^{p}, t_{k l}^{q}+1\right\}=\left\{-t_{k j}^{p} t_{i l}^{q}+t_{k j}^{q} t_{i l}^{p}\right\}
$$

Defining $t_{i j}^{-1}=\delta_{i j}$,

$$
T(z)=\sum_{p \geq-1} t_{i j}^{p} z^{(-p+1)}
$$

where, $T(z)$ generates the centre of infinite Yangian dimension. Incidentally here, the Yang-Baxter equation has no Knots (which were present in Wheeler-Dewitt interpretation of Yang-Baxter) because there is no conformal deformation, there are permutations.

\section{The Standard Model}

\subsection{Elementary Particles}

In Early 20th century, John Dalton first hypothesized about Atoms being a fundamental particle in nature. According to him, atoms were solid balls which were indestructible. Then came Thompson with his electron model. After that his own pupil James Chadwick discovered nucleus also neutron. After the discovery of Nucleons, particle physics took a big jump. Till mid 20th it was thought that electrons are most fundamental particles but then Murray Gillman came up with QUARK model. Till the date, six flavors of quarks are discovered-up, down, charm, strange, top and bottom. Up, charm and top quark have integer charge of $(+2 / 3)$ whereas down, strange and bottom have integer charge of $(-1 / 3)$. All quarks have half integer spin. The Top quark is most recently discovered and is the largest in terms of relativistic mass at $175 \mathrm{GeV} / \mathrm{c}^{2}$. The fundamental constituents of matter are Fermions. Leptons and quarks come under the category of Fermions. There are six flavors of Leptons-electron, muon, tau, electron neutrino, muon neutrino and tau neutrino. All Leptons have half integer spin. Electron, muon and tau have -1 charge spin. Each Lepton has its anti-particle and so do the quarks. Bosons are the Force carriers. The $w(-,+)$ bosons $Z$ bosons and Photons are Gauge Bosons whereas the famous Higgs boson is scalar boson. Graviton (a gravity force mediator) is also a boson but it is not yet discovered (perhaps due to very less interaction 
with matter).

\subsection{Higgs Boson and Higgs Mechanisms}

In 1964, Dr peter Higgs postulated existence of a new type of particle which gives matter mass. Existence of Higgs Boson was confirmed on 4th July 2013 at CERNs LHC. It is because of Higgs field that particles acquire mass. The Higgs field is present in the entire universe as function symmetric field [10]. Higgs mechanism is essential in order to explain property of mass in Gauge Bosons. Higgs field is a $\mathrm{SU}(2)$ doublet pair and hence has 4 real components. All elementary particles are merely excited states (or quanta) of some field. This includes the Higgs boson, which is the quanta of the Higgs field, the photon, which is the quanta of the electromagnetic field, the electron, which is the quanta of the electron field, and so on [10]. All fields exist at all points in time and space. Some fields couple to the Higgs field. After a process called spontaneous symmetry breaking, the Higgs field is separated into two parts. The first part remains a dynamic field, and its quanta are the Higgs bosons. The second part is a constant (called the vacuum expectation value), and the equations that describe the coupling of the Higgs field to other fields become equations that describe the other fields coupling (quadratically) to themselves, which in quantum field theory is interpreted as giving mass to a field. The vacuum expectation value of the Higgs field is therefore proportional to the mass of each field. The vacuum expectation value of the Higgs field is just the value that we would "expect" it to have when it is in its vacuum state, which is the state of lowest energy. It turns out that it is a general law of nature that physical systems always "want" to be in the state of lowest possible energy. The allowed values for the energy are determined by the system's potential energy function. In the case of the Higgs field, the potential function looks (more or less) like Mexican hat topology discussed above [11]. Interaction terms that look like this:

$$
g \bar{\psi} \phi \psi
$$

$\phi$ is the Higgs field. $\psi$ and $\bar{\psi}$ are the fields of some particle and its antiparticle. For example, an electron and a positron. $g$ is just a number, called the coupling constant, which determines how strong the interaction is between the three fields (electron, positron and Higgs). Higgs mechanism can be induced in isotropic manifold as isometry of which fixes a point $p \in g$ (coupling constant) is discrete and is preserved. In order to induce Higgs mechanism in an isotropic Hilbert manifold, it is essential for scalar fields to interact with Dirac fields of type $V \approx g \bar{\psi} \phi \psi($ scalar $) g \bar{\psi} i \gamma^{5} \phi \psi$ (pseudoscalar) This interaction is described as: The action for a meson field $\phi$ interacting with a Dirac baryon field $\psi$ is

$$
S[\phi, \psi]=\int \mathrm{d}^{d}\left[\mathcal{L}_{\text {mesons }} \phi+\mathcal{L}_{\text {dirac }} \psi+\mathcal{L}_{\text {yukawa }}(\phi, \psi)\right]
$$

where the integration is performed over $\mathrm{d}$ dimensions (typically 4 for four-dimensional space time). The meson Lagrangian is given by:

$$
\mathcal{L}_{\text {mesons }}(\phi)=\frac{1}{2} \partial^{\mu} \phi \partial_{\mu}-V(\phi)
$$


Here, $V(\phi)$ is a self-interaction term. For a free-field massive meson, one would have $V(\phi)=\frac{1}{2} \mu^{2} \phi^{2}$ where is the mass of meson. The Yukawa interaction term is:

$$
\mathcal{L}_{\text {Yukawa }}(\phi, \psi)=-g \bar{\psi} \phi \psi
$$

where $g$ is the (real) coupling constant for scalar mesons and $\mathcal{L}_{\text {Yukawa }}(\phi, \psi)=-g \bar{\psi} i \gamma^{5} \phi \psi$ for pseudo scalar mesons. Putting it all together one can write the above more explicitly as:

$$
S[\phi, \psi]=\int \mathrm{d}^{d} x\left[\frac{1}{2} \partial^{\mu} \phi \partial_{\mu} \phi-v(\phi)+\bar{\psi}(i \ddot{d}-m) \psi-g \bar{\psi} \phi \psi\right]
$$

In order to manifest, Higgs field requires spontaneous symmetry breaking which is given by Majorana field Lagrangian. It is also possible to have a Yukawa interaction between a scalar and a Majorana field [12]. The Yukawa interaction involves a scalar and a Dirac spinor which can be thought of as a Yukawa interaction involving a scalar with two Majorana spinors of the same mass as fields exhibit similar geodesics [13]. Broken out in terms of the two chiral Majorana spinors, one has

$$
\begin{aligned}
& S[\phi, \psi] \\
& =\int \mathrm{d}^{d} x\left[\frac{1}{2} \partial^{\mu} \phi \partial_{\mu} \phi-v(\phi)+x^{\Omega} \bar{i} \sigma \cdot \partial x+\frac{i}{2}(m+g \phi) x^{\Gamma} \sigma^{2} x-\frac{i}{2}(m+g \phi) * x^{\Gamma} \sigma^{2} x^{*}\right]
\end{aligned}
$$

where $g$ is a complex coupling constant and $\mathrm{m}$ is a complex number. The Yukawa interaction of a Fermion field $(\psi)$ and Higgs field $(\phi)$ is given as:

$$
\mathcal{L}_{\text {Fermions }}(\phi, A, \psi)=\bar{\psi} \gamma^{\mu} d_{\mu} \psi+G_{\psi} \bar{\psi} \phi \psi
$$

The quantities $\gamma^{\mu}$ are the Dirac matrices, and $G_{\psi}$ is Yukawa coupling protocol. The mass-generation follows the existence of a finite expectation value $\langle|\phi|\rangle$, as seen in Majorana fermion interaction. Again, this is crucial for the existence of the property "mass". As we can see from earlier, Meson field and Higgs field exhibit similar coupling properties. In Abelian Higgs mechanism, scalar field experiences Gauge Invariance i.e. certain gauge transformation does not change energy levels [14]. The Mexican-Hat model very well explains Abelian-Higgs mechanism. The only renormalizable model where a complex scalar field $\phi$ acquires a nonzero value is the Mexican-hat model, where the field energy has a minimum away from zero. The action for this model is:

$$
S(\phi)=\int \frac{1}{2}|\partial \phi|^{2}-\lambda\left(|\phi|^{2}-\Phi^{2}\right)^{2}
$$

which results in the Hamiltonian:

$$
H(\phi)=\frac{1}{2}|\phi|^{2}+|\nabla \phi|^{2}+V(|\phi|)
$$

where $\frac{1}{2}|\phi|^{2}$ shows kinetic energy of Higgs field. From above equation we can deduce the mass exhibited by Higgs field:

$$
\left\langle M^{H \psi}\right\rangle=\frac{2\left[H(\psi)-|\nabla \phi|^{2}-V(|\phi|)\right]}{V \psi^{2}}
$$


where $\psi$ is Dirac-Debroglie matter operator and $V$ is the SMV-operator which is a derivative of Ricci Curvature Tensor.

\subsection{Planckian Scale and Quantum Coupling}

Two Particles are said to be in coupled state when quantum interaction affect both instantaneously at quantum distances. They also undergo changes in quantum states almost instantaneously (almost being the operative word). We can explain changed states as follows:

\subsubsection{Particle 1}

$$
\left\langle\psi_{1}\right\rangle=\frac{1}{\sqrt{2}}(\langle\uparrow \downarrow\rangle-\langle\downarrow \uparrow\rangle)
$$

where $\left\langle\psi_{1}\right\rangle$ is a state vector.

\subsubsection{Particle 2}

$$
\left\langle\psi_{1}\right\rangle=\frac{1}{\sqrt{2}}(\langle\downarrow \uparrow\rangle-\langle\uparrow \downarrow\rangle)
$$

As seen, the eigenstates undergo a small change as in ket notation. This will violate the CKM laws as the wave function will collapse assuming the eigenvalue would be 1 $\mathrm{kg} \cdot \mathrm{m} / \mathrm{s}$. So eliminating the Uncertainty situation, the equations can be combined and modified as:

$$
\left\langle\psi_{1,2}\right\rangle=\frac{1}{\sqrt{2}}(\langle\downarrow+w \uparrow\rangle-\langle\uparrow-w \downarrow\rangle)
$$

where $w$ is wicks expansion of nth order with respect to s-matrix. Substituting all the terms,

$$
\left\langle\psi_{1,2}\right\rangle=\frac{1}{\sqrt{2}}(\langle\downarrow \uparrow\rangle \uparrow\langle\uparrow \downarrow\rangle) \sum_{n=0}^{\infty} \frac{i^{n}}{n !} \int \prod_{j=1}^{n} \mathrm{~d}^{4} x_{j} T \prod_{j=1}^{n} \mathcal{L}_{v}\left(x_{j}\right)=\sum_{n=0}^{\infty} S^{n}
$$

\section{Quantum Relativity}

\subsection{Introduction}

Quantum relativity is like quantum coupling but different in the sense that it also takes dimensionality into consideration. This way, a particle could certainly propagate through space but also a higher dimension as well. Assuming a particle at position $p_{1}$ in space, at time of its decay it is at position $p_{2}$. Today's physics is unable to explain why does the particle propagate the way it does in sub-space topologies? In order to tackle these problems and problems with higher magnitudes, we need to draw a link between the motion of particles and there dimensionalities. By these we can even predict EinsteinRosen bridge [15], get more understanding as on dark matter and dark energy, also may be predict existence of a new particle.

\subsection{Relativistic Quantum Chirality}

Chirality in Quantum physics refers gradual difference in properties between fermions 
and bosons depending on its spin, charge etc. Generally speaking, universe has a preference for left-handed chiral interactions but this does violate the symmetry laws of nature. So there has to be a symmetry maintained if we are to avoid the cp violation. Here comes the quantum approach of symmetry. Talking about Chiral symmetry, Vector gauge theories with mass less Dirac Fermion fields $\psi$ exhibit chiral symmetry, i.e., rotating the left-handed and the right-handed components independently makes no difference to the theory. We can write this as the action of rotation on the fields:

$$
\psi_{L} \rightarrow \mathrm{e}^{\mathrm{i} \theta L} \psi_{L} \psi_{L} \rightarrow \partial \psi_{R} O R \psi_{R} \rightarrow \partial \psi_{L} \psi_{R} \rightarrow \mathrm{e}^{\mathrm{i} \theta R} \psi_{R}
$$

with $N$ flavors, we have unitary rotations instead: $U(N)_{L} X U(N)_{R}$. Assuming 2 mass less quarks $x$ and $y, L=\bar{x} i \varnothing x+\bar{y} i \varnothing y+\mathcal{L}_{\text {Gluon }}$

Now considering Left and right isomorphic spinors

$$
\begin{aligned}
L & =\bar{x}_{L} i \varnothing x_{L}+\bar{x}_{R} i \varnothing x_{R}+\bar{y}_{L} i \varnothing y_{L}+\bar{y}_{R} i \varnothing y_{R}+\mathcal{L}_{\text {Gluon }} \\
q & =\left[\begin{array}{l}
x \\
y
\end{array}\right] \\
L & =\bar{q}_{L} i \varnothing q_{L}+\bar{q}_{R} i \varnothing q_{R}+\mathcal{L}_{\text {Gluon }} \\
L & =\bar{q}_{L} i \varnothing q_{R}+\bar{q}_{R} i \varnothing q_{L}-\mathcal{L}_{\text {Gluon }}
\end{aligned}
$$

where the Gluon field converges. Redefining Left and right anti symmetric chirality:

$$
\begin{aligned}
& \psi_{R}\left(1+\gamma^{5}\right)=\psi_{L}\left(1-\gamma^{5}\right) \\
& \frac{\psi_{R}}{\psi_{L}}=\frac{\left(1-\gamma^{5}\right)}{\left(1+\gamma^{5}\right)}
\end{aligned}
$$

Let, $\frac{\left(1-\gamma^{5}\right)}{\left(1+\gamma^{5}\right)}$ be partial variables for left and right Fermion operator $\check{\mathbb{R}}_{\varnothing i}$. Here $\check{\mathbb{R}}_{\varnothing i}$ is Relativistic proportional operator.

$$
\begin{aligned}
& \check{\mathbb{R}}_{\varnothing i}=\left[\begin{array}{c}
\bar{q}_{L} \\
q_{R}
\end{array}\right] \\
& L=\check{\mathbb{R}}_{\varnothing u} i \varnothing q_{R}+\check{\mathbb{R}}_{\varnothing d} i \varnothing q_{L}-\mathcal{L}_{\text {Gluon }}
\end{aligned}
$$

This briefly implies that chirality depends on how it is observed or from where it is observed. For instance to relate chirality and position with respect to time of two particles $u$ and $d$ :

$$
\begin{aligned}
& \sigma_{x} \sigma_{p} \geq \frac{\hbar}{2} \\
& \sigma_{x}=[x, t] \\
& \sigma_{p}=[m, v] \\
& {\left[\check{\mathbb{R}}_{\varnothing u} i \varnothing q_{R}+\check{\mathbb{R}}_{\varnothing d} i \varnothing q_{L}\right]_{(x, t)} X\left[\check{\mathbb{R}}_{\varnothing u} i \varnothing q_{R}+\check{\mathbb{R}}_{\varnothing d} i \varnothing q_{L}\right]_{(m, v)} \geq \frac{\hbar}{2}} \\
& { }_{(x, t)} X\left[q_{R}+q_{L}\right]_{(m, v)} \geq \frac{\hbar}{2}
\end{aligned}
$$




$$
\begin{gathered}
{\left[\begin{array}{l}
q_{R} \\
q_{L}
\end{array}\right]=\left[\begin{array}{l}
x \\
y
\end{array}\right]} \\
{[x+y]_{(x, t)} X[x-y]_{(m, v)} \geq \frac{\hbar}{2}}
\end{gathered}
$$

From above it can be noted that chirality of particles $u$ and $d$ (taken in the example) differ from chirality observed when at steady state and in motion. It has uncertainty of +2.56 percent. The parity symmetry or in this case anti-symmetry depends on the relativistic proportional operator.

\section{Framework and Scopes of Quantum Relativity}

\subsection{Implications with the Quantum Field Theory}

Quantum Field theory is like a frame work of particles and super particles and also their interactions. It is a mathematical extension of Quantum mechanics. Here every interacting particle is looked upon as interaction of field. Though QFT is promising preposition for standard model completion, it lacks some Kahler geometry due to which gravitons cannot be treated as canonical formalisms of QFT. Properties of gravitons can be predicted by deriving its converging fields in Hilbert space or d-dimensional anti desitter space. We will be using function of stress energy tensor with Hilbert Poyla expansion statistics to determine the exact outcome of properties of Gravitons.

$$
\begin{gathered}
S=\frac{1}{2 k} \int R \sqrt{-g} \mathrm{~d}^{4} x \\
g=\operatorname{det}\left(g_{\mu v}\right) \\
R=g^{\mu v}\left(R_{\mu v}\right) \\
R_{\mu v}-\frac{1}{2} g_{\mu v} R+\Lambda g_{\mu v}=\frac{8 \pi G}{c^{4}} T_{\mu v}
\end{gathered}
$$

Now then, taking stress energy tensor and introducing matrix formulation,

$$
\begin{aligned}
T_{\mu \nu} & =\left[\begin{array}{llll}
T^{00} & T^{01} & T^{02} & T^{03} \\
T^{10} & T^{11} & T^{12} & T^{13} \\
T^{20} & T^{21} & T^{22} & T^{23} \\
T^{30} & T^{31} & T^{32} & T^{33}
\end{array}\right] \\
T_{\mu \nu} & =\frac{-2}{\sqrt{-g}} \frac{\delta \mathcal{L}_{\text {matter }} \sqrt{-g}}{\delta g_{\mu \nu}}
\end{aligned}
$$

so, rearranging the terms and comparing them:

$$
\left[\begin{array}{cccc}
-c^{-2} & 0 & 0 & 0 \\
0 & 1 & 0 & 0 \\
0 & 0 & 1 & 0 \\
0 & 0 & 0 & 1
\end{array}\right]=\frac{-2}{\sqrt{-g}} \frac{\delta \mathcal{L}_{\text {matter }} \sqrt{-g}}{\delta g_{\mu \nu}}
$$




$$
\begin{gathered}
g_{(\alpha, \beta)_{(1,2,3,4)}}=\left[\begin{array}{cccc}
-c^{-2} & 0 & 0 & 0 \\
0 & 1 & 0 & 0 \\
0 & 0 & 1 & 0 \\
0 & 0 & 0 & 1
\end{array}\right] \\
\left(g^{\alpha \beta}\right)=\frac{-2}{\sqrt{-g}} \frac{\delta \mathcal{L}_{\text {matter }} \sqrt{-g}}{\delta g_{\mu v}} \\
g=g^{\mu v} \mathrm{~d} x^{\alpha} \otimes \mathrm{d} x^{\beta}
\end{gathered}
$$

The factors $\mathrm{d} x^{\alpha}, \mathrm{d} x^{\beta}$ are one-form gradients of the scalar coordinate fields $g^{\mu \nu}$. The metric is thus a linear combination of tensor products of one-form gradients of coordinates. The coefficients are a set of 16 real-valued functions (since the tensor $g$ is actually a tensor field, which is defined at all points of a space time manifold). In order for the metric to be symmetric we must have

$$
\begin{aligned}
& g^{\alpha \beta}=g^{\mu v} \\
& g^{\mu v}=\frac{-2}{\sqrt{-g}} \frac{\delta \mathcal{L}_{\text {matter }} \sqrt{-g}}{\delta g_{\mu v}}
\end{aligned}
$$

As the Lagrangian is nth dimensional gradient of the field, the delta function has no real value and can be therefore eliminated,

$$
\mathcal{L}_{\text {matter }}=-\frac{1}{2}
$$

By using Quantum relativity, we can derive the following conclusion:

$$
S\left[\varphi_{i}\right]=\int \mathcal{L}_{\text {matter }}\left(\varphi_{i}(S), \frac{\gamma \varphi_{i}(S)}{\gamma S^{\alpha}}, S^{\alpha}\right) \mathrm{d}^{n} S
$$

$S$, is a functional of the dependent variables $\varphi_{i} S$ with their derivatives and $s$ itself. where $S=S^{\alpha}$ denotes the set of $n$ independent variables of the system, indexed by $\alpha=1,2,3, \cdots, n$. Notice $L$ is used in the case of one independent variable $(t)$ and is used in the case of multiple independent variables (usually four: $x, y, z, t$ ).

$$
\begin{aligned}
& S[\varphi]=\int L\left(\varphi, \nabla \varphi, \frac{\partial \varphi}{\partial t}, x, t\right) \mathrm{d}^{3} x \mathrm{~d} t \\
& \int \nabla \varphi^{x} \varphi^{y} \varphi^{z} \varphi^{t}=\int-\frac{1}{2}\left(\varphi_{i}(S), \frac{\gamma \varphi_{i}(S)}{\gamma S^{\alpha}}, S^{\alpha}\right) \mathrm{d}^{n} S \\
& \nabla \varphi^{x} \varphi^{y} \varphi^{z} \varphi^{t}=-\frac{1}{2}\left(\varphi_{i}(S), \frac{\gamma \varphi_{i}(S)}{\gamma S^{\alpha}}, S^{\alpha}\right) \mathrm{d}^{n} S
\end{aligned}
$$

Let $\nabla \varphi^{x} \varphi^{y} \varphi^{z} \varphi^{t}$ be spatial matter operator $\left(m^{\nabla \varphi^{x} \varphi^{y} \varphi^{z} \varphi^{t}}\right)$ in 4 dimensional space time. Four dimensions are considered as a result of Kaluza Klein manifolds exerted by d-dimensional de-sitter space in $n=11$ string theory. Moreover time is a radical component of space time and thus gravity too.

$$
\left(m^{\nabla \varphi^{x} \varphi^{y} \varphi^{z} \varphi^{t}}\right)=-\frac{1}{2}\left(\varphi_{i}(S), \frac{\gamma \varphi_{i}(S)}{\gamma S^{\alpha}}, S^{\alpha}\right) \mathrm{d}^{n} S
$$


This conclusively proves Gravitons (assuming gravitons are 1 dimensional entity but can traverse through all 4 dimensions) have some rest mass:

$$
\left(m^{1,2,3,4}\right)=-\frac{1}{2}\left(\varphi_{i}(S), \frac{\gamma \varphi_{i}(S)}{\gamma S^{\alpha}}, S^{\alpha}\right) \mathrm{d}^{n} S
$$

\subsection{A New Particle? Using the Hubbard Stratonovich Transformation Using Auxilliary Fields to Predict a Quasi Particle}

Axiomatically speaking, Graviton and Gravitino being super partners of each other interact with each other. Since as proved earlier that Gravitons posses some mass, we can imply Relativistic Dirac-Schrodinger equation to interact with Rarita-Schwinger equation:

$$
\left(\varepsilon^{\mu \kappa \rho \nu} \gamma_{5} \gamma_{\kappa} \partial_{\rho}-i m \rho^{\mu \nu}\right) \psi_{v}=0 \Rightarrow\left(\frac{i \hbar}{2 m} \psi^{*} \partial^{\mu} \psi-\psi \partial^{\mu} \psi^{*}\right)
$$

$\varepsilon^{\mu \kappa \rho \nu}$ is the Levi-Civita symbol, $\gamma_{5}$ and $\gamma_{\mu}$ are Dirac matrices, is the mass, $\rho^{\mu \nu}=\frac{i}{2}\left[\gamma^{\mu}, \gamma^{v}\right]$, and $\psi_{v}$ is a vector spinor with additional components compared to the four component spinor in the Dirac equation. Interaction produces an auxiliary field. Thus it follows Hubbard Stratonovich transformation. This aux field particle could be a new type of Trion as it posses $3 / 2$ lie spin. Let it be called G-boson. Lagrangian can be derived as:

$$
F^{\alpha}=\left(\Gamma_{\beta c}^{\alpha} \frac{\mathrm{d} \xi^{\beta}}{\mathrm{d} t} \frac{\mathrm{d} \xi^{c}}{\mathrm{~d} t}\right), \xi^{\alpha}=\frac{\mathrm{d} \xi^{a}}{\mathrm{~d} t}
$$

Here $F^{\alpha}$ is the $a^{\text {th }}$ contra variant components of the resultant force acting on the particle, $\Gamma_{\beta c}^{\alpha}$ are the Christoffel symbols of the second kind. The kinetic energy of particles can be derived as:

$$
T=\frac{1}{2} m g_{\beta c} \frac{\mathrm{d} \xi^{\beta}}{\mathrm{d} t} \frac{\mathrm{d} \xi^{c}}{\mathrm{~d} t}
$$

$g_{\beta c}$ is the covariant components of the metric tensor of the hyperbolic coordinate system. All indices each take the values 1, 2, 3. Hyperbolic coordinates are not the same as generalized coordinates.

\subsection{Quantum Relativity and Dark Energy Models}

Various cosmological models state existence of dark energy as apparent reason for expansion of universe [16]. The Lambda-CDM model is prominent in relating special relativity and inflationary cosmology. It is a parameterization of the Big Bang cosmological model in which the universe contains a cosmological constant, denoted by Lambda (Greek), associated with dark energy, and cold dark matter (abbreviated CDM). It is frequently referred to as the standard model of Big Bang cosmology. The other favorable model is the CHGS model. The Callan Giddings Harvey Strominger model or CGHS in short is a toy model of general relativity in 1 spatial and 1 time dimension. General relativity is a highly nonlinear model, and as such, its $3+1 \mathrm{D}$ version is usually 
too complicated to analyze in detail. In $3+1 \mathrm{D}$ and higher, propagating gravitational waves exist, but not in $2+1 \mathrm{D}$ or $1+1 \mathrm{D}$. In $2+1 \mathrm{D}$, general relativity becomes a topological field theory with no local degrees of freedom, and all $1+1 \mathrm{D}$ models are locally flat. These models even though explain other cataclysmic phenomena, they are more than useless to tamper with dark energy. Point to be noted is that Lamda-CDM model tries to explain Dark energy by virtue of Chameleon particle, a hypothetical scalar particle that couples to matter more weakly than gravity. By applying Quantum relativity, dark energy can be explained as follows: Assuming the following things:

1) Space is $5 \mathrm{D}$ i.e. 4 spatial dimensions $+1 \mathrm{D}$ time.

2) All particles are invariant under gravity.

3) Universe is expanding BUT has boundaries.

4) Dark energy exists.

There is supposedly a particle, randomly placed in spatial dimensions. It obeys Quantum chaotic distribution and is in highly excited state. Let the initial position of particle be $P_{1}$ and final be $P_{2}$. By using QR (quantum relativity),

$$
\left[\bar{\phi}_{D E}\right] \approx P_{1}\left[x, y, z, t,-t_{0}\right] \Rightarrow P_{2}\left[x, y, z, t,-t_{0}\right]
$$

This is condensing phasor equation for initial position of particle and its divergence with respect to final position. Here, $\left[\bar{\phi}_{D E}\right]$ is canonical formalism of Dark energy spread uniformly through five dimensions, $-t_{0}$ is time coordinate at beginning of expansion of universe. Now after effect of dark energy on the particle,

$$
\left[\bar{\phi}_{D E}\right] \approx-P_{0}\left[x, y, z, t,-t_{0}\right]+P_{1}\left[x, y, z, t,-t_{0}\right] \Rightarrow P_{2}\left[x, y, z, t,-t_{0}\right]
$$

Here, $-p_{0}$ is the position of particle at instant before dark energy acts on it, $+P_{1}$ is the position of particle at instant after dark energy acts on it. For that particular instant of time, the particle exists at initial as well as final position simultaneously and in initial state and excited state simultaneously before finally showing presence at $p_{2}$ in initial quantum state. This can also mean that at that particular instant of time, the particle temporarily decays into 2 particles before finally becoming a single again. Derivating for that particular time instant:

$$
\prod T=\int_{-n}^{n} \delta\left[\bar{\phi}_{D E}\right]-\approx\left[\bar{\phi}_{D E}\right] \stackrel{\text { Yields }}{\longrightarrow} P_{2}\left[x, y, z, t,-t_{0}\right] \mathrm{d} n
$$

\subsection{Banach-Tarski Theorem Solution}

The Banach-Tarski paradox is a theorem in set-theoretic geometry [17], which states the following: Given a solid ball in 3 dimensional space, there exists a decomposition of the ball into a finite number of disjoint subsets, which can then be put back together in a different way to yield two identical copies of the original ball. Indeed, the reassembly process involves only moving the pieces around and rotating them, without changing their shape. However, the pieces themselves are not "solids" in the usual sense, but infinite scatterings of points. It is yet unproven hypothesis. Using condensing phasor equation: 


$$
\left[\phi_{F}\right] \cong \sum_{\infty}^{1}(x, m) \Rightarrow \sum_{1}^{\infty}\left(x_{n}, m_{n}\right)
$$

Here, $\left[\phi_{F}\right]$ is the breaking force for an idealized sphere [18]. The above relation specifies the quantum superposition of $\mathrm{n}$ number of particles condensed to one instant before breaking and split into infinite number of particles and grouping together to form infinite number of idealised spheres. This is non-algebraic proof of Banach-Tarski theorem by Quantum relativity.

\section{Conclusion}

To conclude this paper, we have discussed many things through. In Section 2, we thoroughly discussed Quantum Field Theory and saw how physics behaves differently at atomic level. In Section 3, we saw the grand unification theory and revisited that yang-baxter equation forms knots. In Section 4, we saw Higgs-mechanism. We also proved that Mass is produced by virtue of kinetic energy of Higgs field thus eliminating the possibility that Higgs boson and Higgs-field is mass less. We solved the quantum coupling problem by formalizing Wick rotation and canonical invariance. Turning to most important section in this paper, first I described the general principle of Quantum relativity and then derived its formula for chiral and achiral symmetries. I also proved that chirality exists in standard model i.e. the result of collision of particles depends on how and where it is observed instead of it being and obsolete thing. In Section 6, we proved that gravitons do posses certain mass. We predicted existence of a new particle (theoretically speaking) by the presence of auxiliary field decay of graviton-gravitino interaction. We also related quantum relativity to dark matter and proved that dark energy is nothing but extra manifold with hinge of accelerated time reference. We also developed a new way to relate the position and momenta of a single particle before and after decay known as condensation phasor equation. Lastly, we solved Banach-Tarski theorem by employing this new method and QR.

\section{References}

[1] Greene, B.R. and Plesser, M.R. (1990) Duality in Calabi-Yau Moduli Space. Nuclear Physics B, 338, 15-37. http://dx.doi.org/10.1016/0550-3213(90)90622-K

[2] Brihaye, Y. and Delsate, T. (2007) Charged-Rotating Black Holes and Black Strings in Higher Dimensional Einstein-Maxwell Theory with a Positive Cosmological Constant. Classical and Quantum Gravity, 24, 4691. http://dx.doi.org/10.1088/0264-9381/24/18/010

[3] Chen, X., Donaldson, S. and Sun, S. (2015) K“"ahler-Einstein Metrics on Fano Manifolds. I: Approximation of Metrics with Cone Singularities. Journal of the American Mathematical Society, 28, 183-197. http://dx.doi.org/10.1090/S0894-0347-2014-00799-2

[4] Thorn, J.J., Neel, M.S., Donato, V.W., Bergreen, G.S., Davies, R.E. and Beck, M. (2004) Observing the Quantum Behavior of Light in an Undergraduate Laboratory. American Journal of Physics, 72, 1210-1219. http://dx.doi.org/10.1119/1.1737397

[5] Born, M., Heisenberg, W. and Jordan, P. (1926) Zur Quantenmechanik. II. Zeitschrift fur Physik, 35, 557-615. http://dx.doi.org/10.1007/BF01379806

[6] Wick, G.C. (1954) Properties of Bethe-Salpeter Wave Functions. Physical Review, 96, 1124- 
1134. http://dx.doi.org/10.1103/PhysRev.96.1124

[7] Le Bellac, M., Barton, G., et al. (1991) Quantum and Statistical Field Theory. Clarendon Press, Oxford.

[8] Georgi, H. and Glashow, S.L. (1974) Unity of All Elementary-Particle Forces. Physical Review Letters, 32, 438-441. http://dx.doi.org/10.1103/PhysRevLett.32.438

[9] Dewitt, B.S. (1967) Quantum Theory of Gravity. I. The Canonical Theory. Physical Review, 160, 1113-1148. http://dx.doi.org/10.1103/PhysRev.160.1113

[10] Chung, D.-Y. (2015) The Big Bang Started by the Creation of the Reverse Higgs Field. Journal of Modern Physics, 6, 1189-1194. http://dx.doi.org/10.4236/jmp.2015.69123

[11] Da Silva Filho, J.I. (2016) Undulatory Theory with paraconsistent Logic (Part I): Quantum Logical Model with Two Wave Functions. Journal of Quantum Information Science, 6, 143. http://dx.doi.org/10.4236/jqis.2016.63012

[12] Majorana, E. and Maiani, L. (2006) A Symmetric Theory of Electrons and Positrons. Ettore Majorana Scientific Papers, Springer, Berlin, 201-233. http://dx.doi.org/10.1007/978-3-540-48095-2 10

[13] Keung, W.-Y. and Senjanovi'c, G. (1983) Majorana Neutrinos and the Production of the Right-Handed Charged Gauge Boson. Physical Review Letters, 50, 1427-1430. http://dx.doi.org/10.1103/PhysRevLett.50.1427

[14] El Naschie, M.S. (2016) Einstein-Rosen Bridge (ER), Einstein-Podolsky-Rosen Experiment (EPR) and Zero Measure Rindler-Kamcantorian Spacetime Geometry (ZMG) Are Conceptually Equivalent. Journal of Quantum Information Science, 6.

[15] Rigden, J.S. (2009) Einstein: His Life and Universe by Walter Isaacson. Historian, 71, 158159.

[16] Gross, D.J., Harvey, J.A., Martinec, E. and Rohm, R. (1985) Heterotic String Theory (I). The Free Heterotic String. Nuclear Physics B, 256, 253-284. http://dx.doi.org/10.1016/0550-3213(85)90394-3

[17] Churkin, V.A. (2010) A Continuous Version of the Hausdor-Banach-Tarski Paradox. Algebra and Logic, 49, 91-98. http://dx.doi.org/10.1007/s10469-010-9080-y

[18] Gubser, S.S., Klebanov, I.R. and Polyakov, A.M. (1998) Gauge Theory Correlators from Non-Critical String Theory. Physics Letters B, 428, 105-114. http://dx.doi.org/10.1016/S0370-2693(98)00377-3 
Submit or recommend next manuscript to SCIRP and we will provide best service for you:

Accepting pre-submission inquiries through Email, Facebook, LinkedIn, Twitter, etc.

A wide selection of journals (inclusive of 9 subjects, more than 200 journals)

Providing 24-hour high-quality service

User-friendly online submission system

Fair and swift peer-review system

Efficient typesetting and proofreading procedure

Display of the result of downloads and visits, as well as the number of cited articles

Maximum dissemination of your research work

Submit your manuscript at: http://papersubmission.scirp.org/

Or contact iqis@scirp.org 